Sādhanā, Vol. 18, Parts 3 \& 4, August 1993, pp. 683-694. (C) Printed in India.

\title{
Numerical study of bluff body flow structures
}

\author{
GUOCAN LING
}

Laboratory for Nonlinear Mechanics, Institute of Mechanics, Chinese Academy of Sciences, Beijing, 100080 , China

\begin{abstract}
Our recent progress in numerical studies of bluff body flow structures and a new method for the numerical analysis of near wake flow field for high Reynolds number flow are introduced. The paper consists of three parts. In part one, the evolution of wake vortex structure and variation of forces on a flat plate in harmonic oscillatory flows and in in-line steady-harmonic combined flows are presented by an improved discrete vortex method, as the Keulegan-Carpenter number $(\mathrm{KC})$ varies from 2 to 40 and ratios of $U_{m}$ to $U_{0}$ are of $O\left(10^{-1}\right), O(1)$ and $O(10)$, respectively. In part 2 , a domain decomposition hybrid method, combining the finite-difference and vortex methods for numerical simulation of unsteady viscous separated flow around a bluff body, is introduced. By the new method, some high resolution numerical visualization on near wake evolution behind a circular cylinder at $\operatorname{Re}=10^{2}, 10^{3}$ and $3 \times 10^{3}$ are shown. In part 3 , the mechanism and the dynamic process for the three-dimensional evolution of the Kármán vortex and vortex filaments in braid regions as well as the early features of turbulent structure in the wake behind a circular cylinder are presented numerically by the vortex dynamics method.
\end{abstract}

Keywords. Near wake evolution; hybrid method; three-dimensional evolution; turbulent structure; bluff body.

\section{Introduction}

Bluff body flow has received a great deal of attention especially for the prediction of loads on engineering structures such as offshore platform supports, ocean pipelines, risers, high buildings, bridge piers etc. and for analysis of wake control in aeronautical engineering as well. Of special interest, from a more fundamental point of view, are recent investigations of flow structures from bluff cylinders in incompressible flows, which may be classified into three aspects. (1) The complex flow structure from an oscillating cylinder, which contains different modes of vortex formation around the cylinder as the Keulegan-Carpenter (KC) number varies; mode competition when the cylinder is subjected to forced oscillations in a steady stream with varying frequency and amplitude or in a different direction with respect to the steady flow; vortex lock-in etc. In these cases, the flow development and the force variation on the cylinder will exhibit complicated nonlinear behaviour. (2) The high Reynolds number separated 
flow about the bluff body, such as the long time behaviour of vortex structures containing different scales near and behind the cylinder, the interaction among secondary vortices and the primary vortex, the mechanism of vortex shedding, flow instability and transition. (3) The three dimensionality and coherent structures in the wake of two-dimensional bluff bodies, which cover the mechanism of 3-D development of wake flow, the different types of large structures in near-wake, intermediate-wake and far-wake, respectively, the topological structure and the 3-D spatial organizations of vorticity, and the driving mechanism responsible for the growth of these large structures. In the last ten years, numerous research efforts have led to a better insight into some of the relevant mechanisms referred to above [e.g. for the first aspect, see Sarpkaya (1986), Obasaju et al (1988) and Ongoren \& Rockwell (1988); for the second, see Bouard \& Coutanceau (1980) and Braza et al (1986); and for the third, see Okubo et al (1988) and Williamson $(1986,1992)$; owing to paucity of space a complete review is not given here]; however, there are a number of interesting features of bluff body flow that have largely remained unexplored. Moreover, most of the previous studies are concerned with experiments. It is believed that further study is needed, especially by numerical simulation. Concerning this point, there are crucial difficulties in the numerical analysis of high Reynolds number flow, containing detailed simulation of vortex structure evolution with different scales and flow transition in near wake of cylinders, and for flow field analysis of oscillating flow over cylinders, where flow transition occurs and Reynolds numbers may range from subcritical to supercritical, as well as for the three-dimensional transition of plane wake flow and computation of coherent structures. Hence it is desirable to develop a more effective numerical method which will reduce the difficulty in finite-difference solutions of Navier-Stokes $(\mathrm{N}-\mathrm{S})$ equations at high Reynolds numbers and overcome the theoretical weakness of the vortex method which fails in predicting accurately the flow field near a vorticity layer and body surface. The new method should not only be capable of precisely predicting the small structures near the body but also yield satisfactorily the global feature of vortex shedding flow. Besides, further effort should be made using some improved vortex (vortex filaments) method based on inviscid vortex dynamics, to study the global features of bluff body flow and the character of early three-dimensional transition of the wake flow.

In this paper, our recent progress in numerical studies of bluff body flow structure and a new method for numerical analysis of the near-wake flow field for high Reynolds number separated flow are introduced. New results and findings on the evolution of vortex patterns, variation of force, detailed behaviours of flow structure behind a flat plate and a circular cylinder in steady and steady-harmonic flow, as well as some new insights into the mechanism of three-dimensional evolution of wake vorticity structure are reported. The paper is in three sections.

\section{Evolution of wake structures and variation of forces of a flat plate}

In this section, results on the evolution of wake structure and variation of the forces on a flat plate in harmonic oscillatory flows and in in-line steady-harmonic combined flows are presented numerically by an improved discrete vortex method (Ling \& Luo 1991; Ling \& Liu 1992). For the oscillatory oncoming flow case, our calculations present a new mode of vortex motion in the wake of the plate for low $\mathrm{KC}$ number $(\mathrm{KC}<5)$ describing vortex shedding, pairing and moving in a period of the oscillatory flow starting from rest, as shown in figure 1 . That is, during the wake reversal, the 

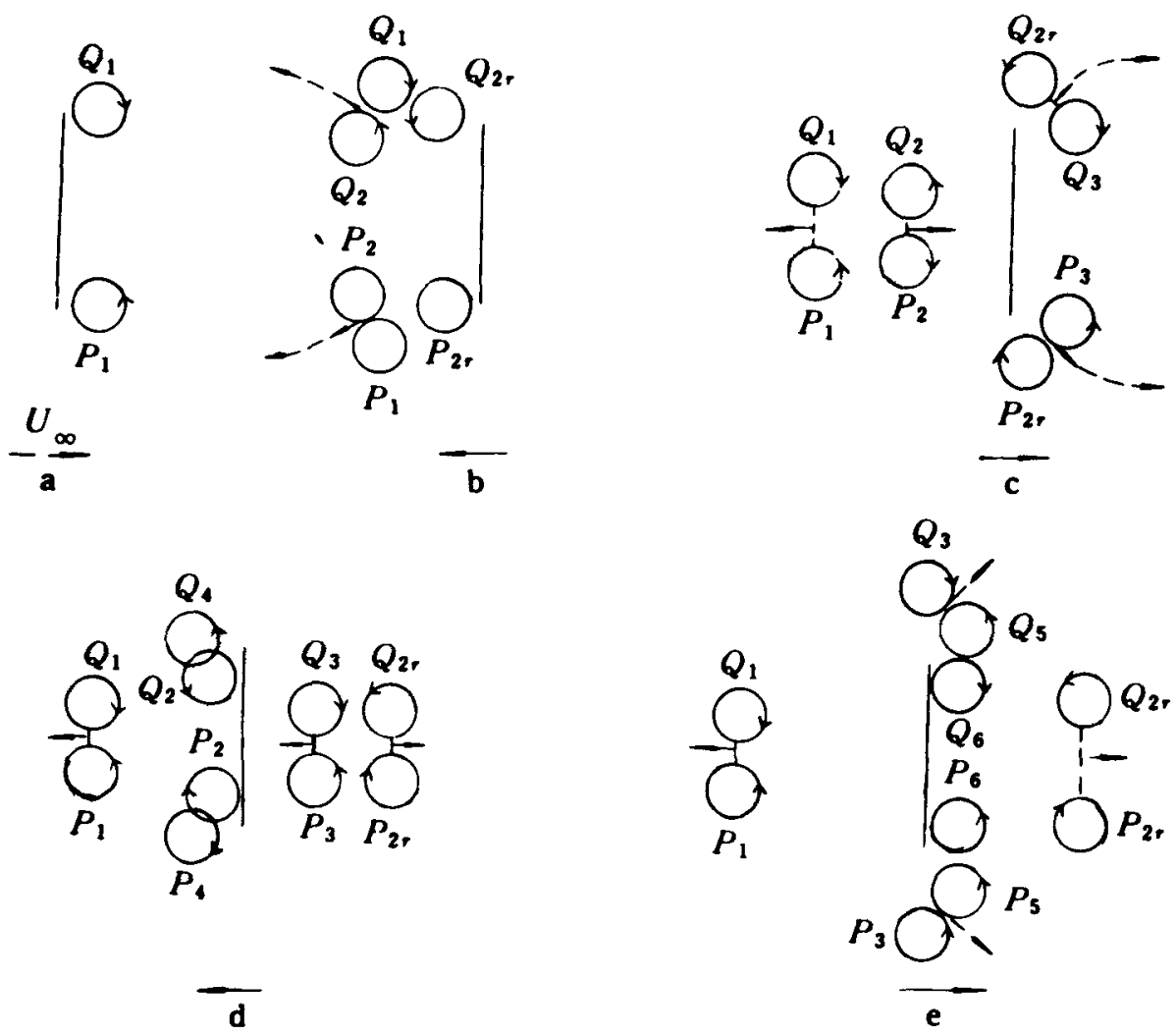

Figure 1. Scheme of vortex motion.

vortices are periodically paired and re-paired; the movements of paired vortices are regular; a pair of vortices moves away from the plate while another comes close to the plate, the phenomenon occurring on both sides of the plate with a phase difference. For long time behaviour of the vortex structure, when the $\mathrm{KC}$ number varies from 2 to 40 , the vortex pattern changes from horizontal distributed vortex cluster in the range of small KC numbers to a slightly inclined "harmonic wave" shaped vortex-cloud in the range of moderate $\mathrm{KC}$ numbers, then becomes a vortex cluster inclined at an angle of nearly $50^{\circ}$ to the oncoming flow at $\mathrm{KC}=20$, and finally, as $\mathrm{KC}$ number increases still further, it is like a normal Kármán vortex street. The evolution of vortex pattern with $\mathrm{KC}$ number is shown in figure 2 . The predicted drag and inertia force coefficients are closer to the experimental results of Keulegan \& Carpenter (1958) as compared with previous vortex simulation by other authors (see figures 3 and 4). The existence of a minimum inertial force coefficient near $\mathrm{KC}=20$ is also well predicted and the reason for this phenomenon has been given through an analysis of the vortex structure. For a steady oncoming flow case, the calculated global features of the separated flow, the variations of time-averaged longitudinal velocity component, the turbulence intensity $\left(\bar{u}^{\prime 2}\right)^{1 / 2} / U_{\infty}$, and the Reynolds stress $-\overline{u^{\prime} v^{\prime}} / U_{\infty}^{2}$ in the wake, are in fair agreement with or close to previous vortex simulation or experimental measurements, respectively. Some examples are shown in figure $5(\mathrm{a}-\mathrm{c})$. For the steady-harmonic combined oncoming flow case, there are three vortex modes, namely a vortex street, a "longitudinal wave" shaped vortex-cloud, and a vortex cluster structure corresponding to three typical in-line combinations of oncoming flow components, i.e. the ratios $U_{m}$ to $U_{0}$, which are $O\left(10^{-1}\right), O(1)$ and $O\left(10^{1}\right)$, 

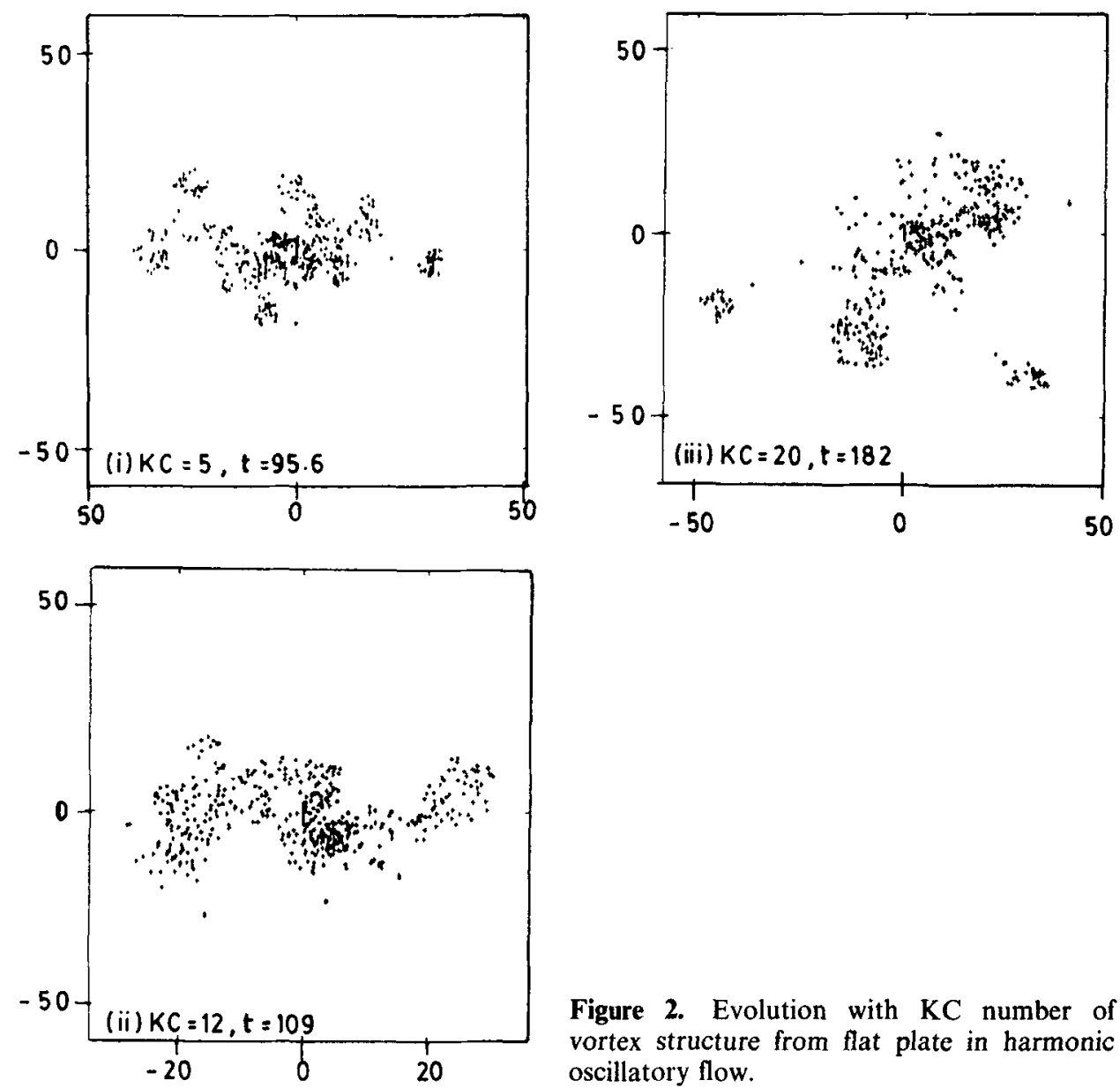

Figure 2. Evolution with $\mathrm{KC}$ number of vortex structure from flat plate in harmonic oscillatory flow.

respectively. Some examples are shown in figure 6. The variation of forces on and vorticity shedding rate from the plate in the combination flow are obtained. The effect on the prediction of forces on the plate and vorticity shedding rate from the disturbance component in combined flow is demonstrated quantitatively and

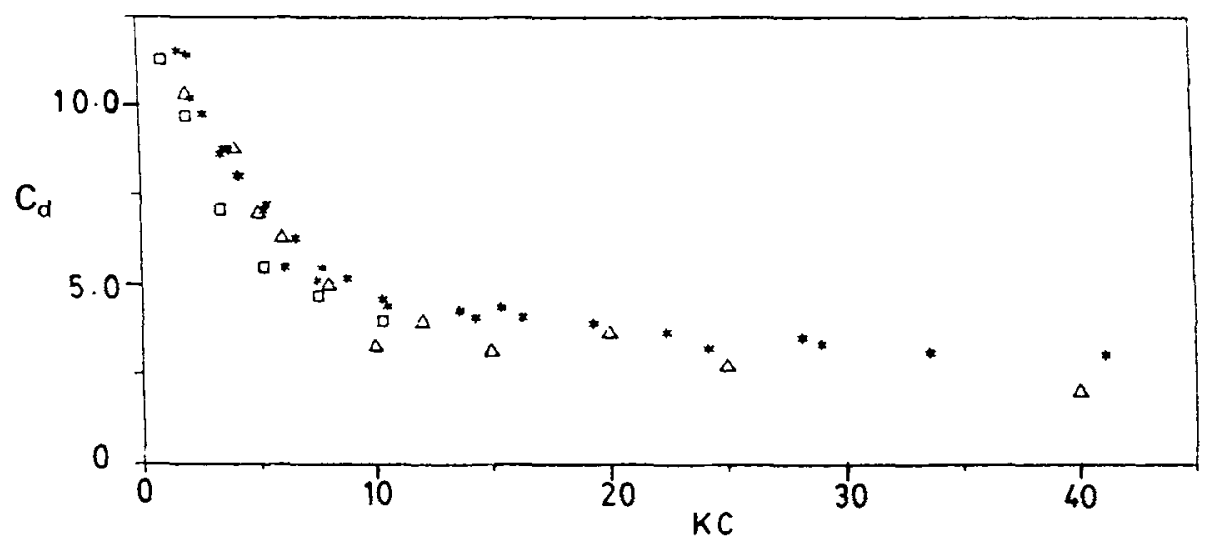

Figure 3. Variation with $\mathrm{KC}$ number of drag coefficient for flat plate. $[\Delta-$ Present results; *- Keulegan \& Carpenter (1958); $\square$ - Lian (1980).] 


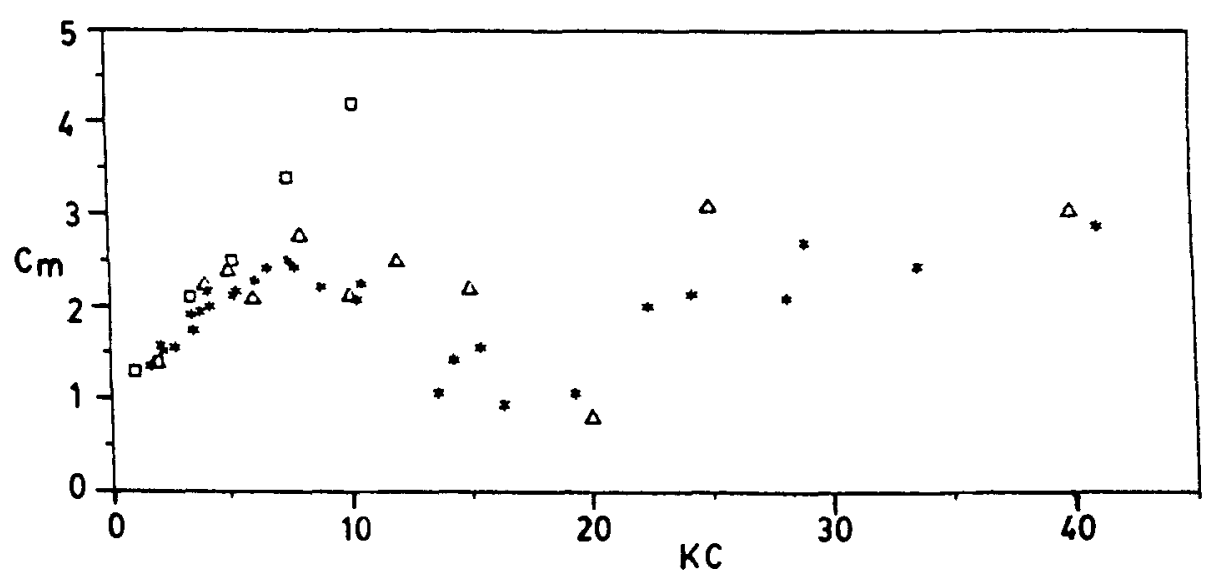

Figure 4. Variation with $\mathrm{KC}$ number of inertia force coefficient for flat plate. [ $\Delta$ - Present results; * - Keulegan \& Carpenter (1958), $\square$ - Lian (1980).]

analysed. In general, superposing a steady flow as a disturbance component on oscillatory oncoming flow makes the mean value of drag coefficient decrease, and vice versa, imposing an oscillatory flow on steady oncoming flow increases the drag and vorticity shedding rate; especially in the small $\mathrm{KC}$ number case the disturbance has considerable influence, but there is no big influence on the inertia force coefficient. The vortex lock-in phenomenon has been simulated. From our calculations for parameters $\mathrm{KC}=2-8, U_{m} / U_{0}=0.8-10$, a correlation of drag coefficient with $\mathrm{KC}$ and $U_{m} / U_{0}$ has been found, namely

or

$$
C_{d}^{2}=17 \cdot 7\left[(1 / \mathrm{KC}) \cdot\left(U_{m} / U_{0}\right)\right]+0 \cdot 105
$$

$$
C_{d}^{2}=17 \cdot 7 V_{r}^{-1}+0.105
$$

where $V_{r}$ is reduced velocity. The corresponding curve is shown in figure 7.

\section{Domain decomposition hybrid method and numerical visualization of near-wake flow structure behind a circular cylinder}

In order to reduce the difficulties in the finite-difference solutions for $\mathrm{N}-\mathrm{S}$ equations for high Reynolds number bluff body flows and to overcome the theoretical weakness of the vortex method, which is unable to predict precisely the small scale structure near the body, while taking advantage of both methods, a domain decomposition hybrid method combining the finite-difference and vortex methods has now developed (Ling et al 1992b). The full flow field is decomposed into an interior domain $\Omega 1$ near the body surface and an exterior domain $\Omega 2$, and an interface. The extent of the interior domain is $O(R)(R$ is a characteristic length of the body). In the domain $\Omega 1$, the flow is viscous and computed by a finite-difference scheme based on the N-S equations and the Poisson stream function equation. In $\Omega 2$, the flow is regarded approximately as an inviscid potential flow. The convection of vortices is calculated by a discrete vortex model using the vortex-in-cell method. The flows in the two domains are coupled and solved simultaneously. The boundary values of the vorticity on the interface required for solving the vorticity transport equation in $\Omega 1$ are given 

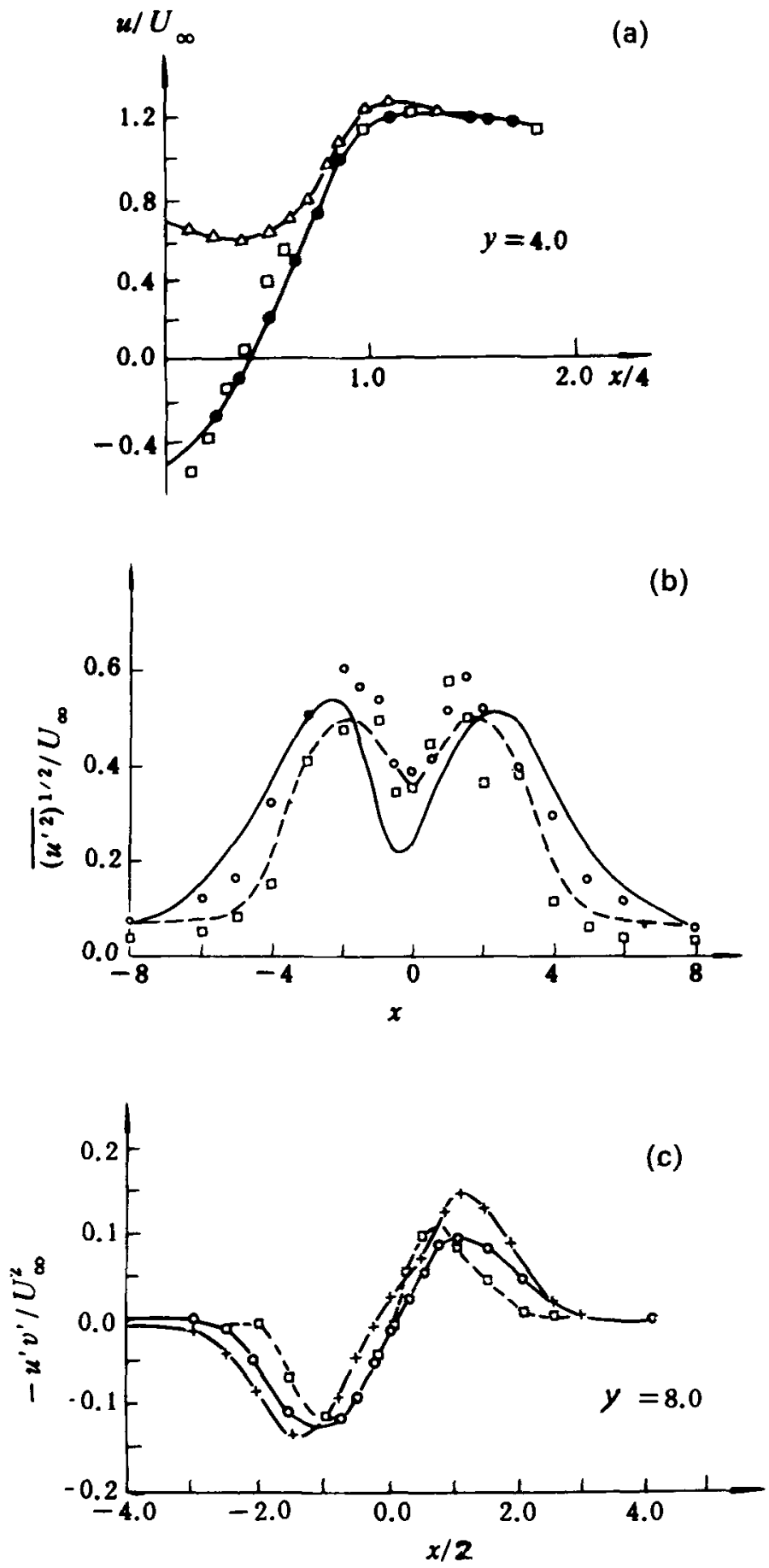

Figure 5. (a) Time-averaged longitudinal velocity component in the wake $\left(\alpha=90^{\circ}\right)$; $\circ-$ present results, $-\Delta,-\square$ Bradbury (1976). (b) Time-averaged longitudinal turbulent intensity (at $y=4 \cdot 0$ ), - present results, -.-Bradbury (1976), $\square$ Kiya \& Arie (1980), o Chein \& Chung (1988). (c) Time-averaged Reynolds stress in the wake $\left(\alpha=20^{\circ}\right),-+-$ present results, $-\circ-$ Chein \& Chung (1988), $-\square$ Kiya \& Arie (1980). 

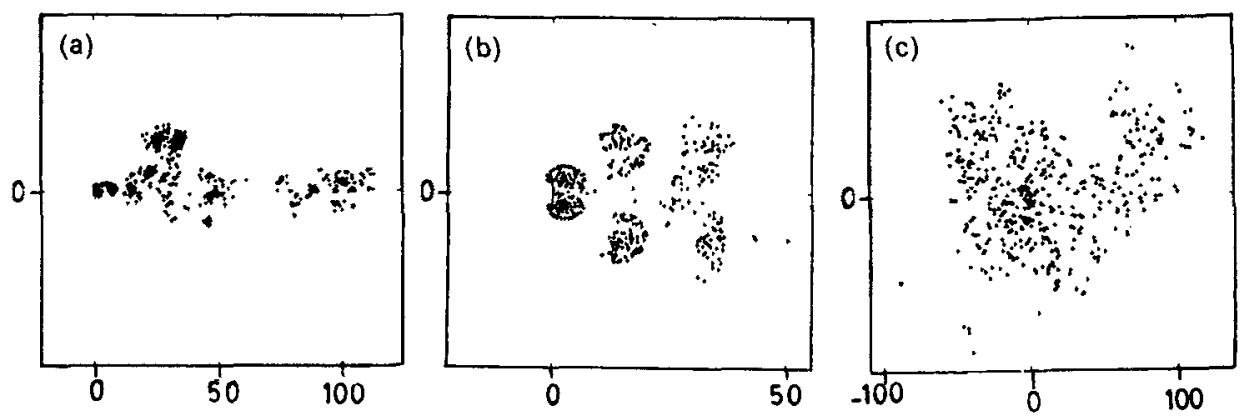

Figure 6. Vortex patterns over long times in the wake of flat plate in steadyharmonic flows. (a) $U_{m} / U_{0}=0 \cdot 5, t=159 \cdot 3, \mathrm{KC}=10$; (b) $U_{m} / U_{0}=1 \cdot 0, t=42 \cdot 0$, $\mathrm{KC}=4 ;$ (c) $U_{m} / U_{0}=10, t=79 \cdot 2, \mathrm{KC}=40$.

carefully. Matching conditions for the flow in the two domains are established and numerical schemes for the hybrid method are described in detail (Ling et al 1992b).

As an application of the present method, numerical solutions for flows around a circular cylinder at $\operatorname{Re}_{\infty}=100,1000$ and 3000 are given in detail. For $\operatorname{Re}=3 \times 10^{3}$, the calculated flow structures are in excellent agreement with the experimental results given by Bouard \& Coutanceau (1980). In addition some new features of the complex flow structure at long times have been discovered for the first time, using a small interior domain, a second-order accurate scheme and very small mesh size. The calculated results show that in the near wake there are three kinds of vortex structures

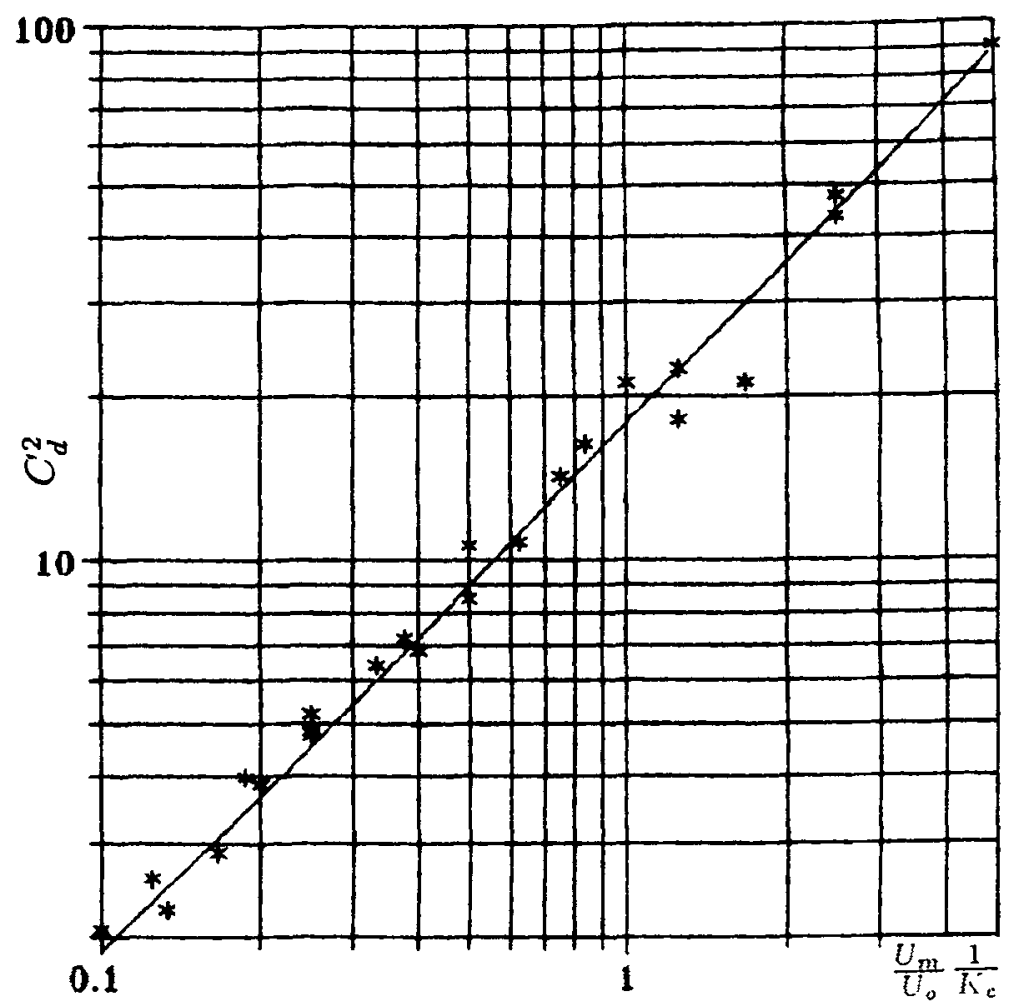

Figure 7. $C_{d}^{2}$ vs $\left(U_{m} / U_{0}\right) / \mathrm{KC}$ for a flat plate in steady-harmonic flow. 
(a)

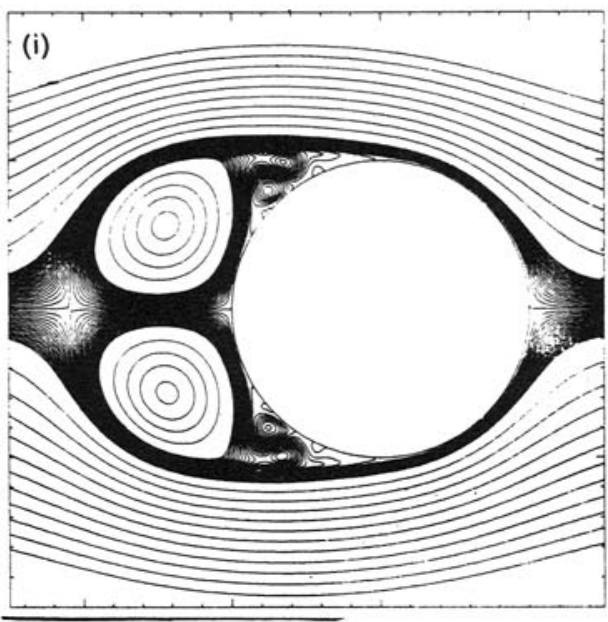

(b)
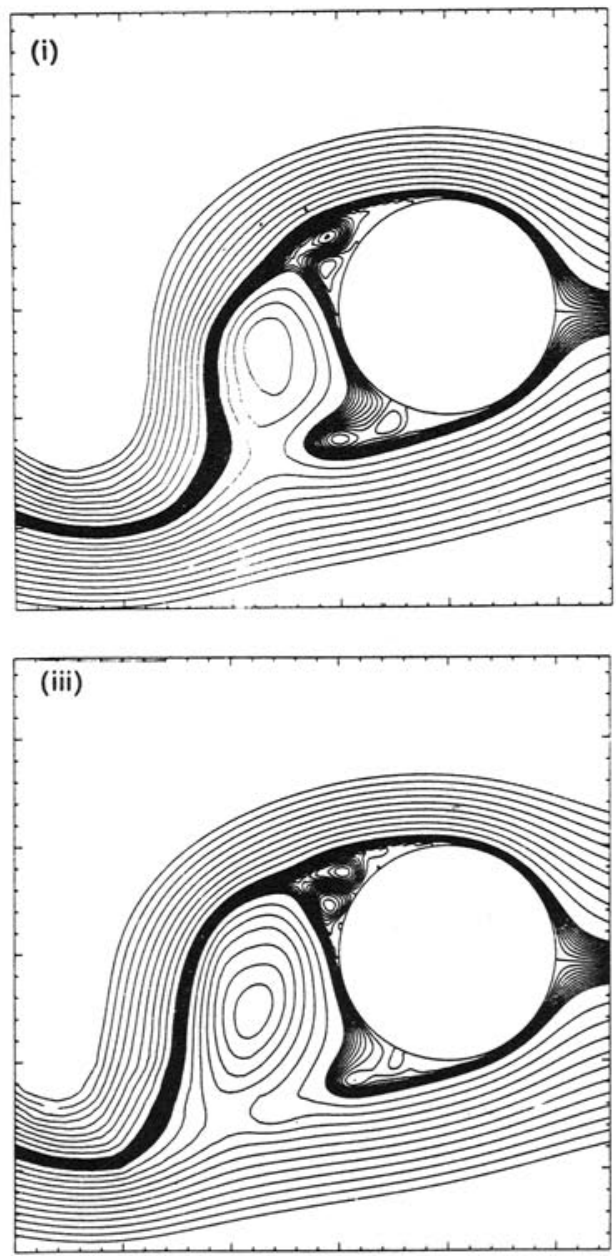

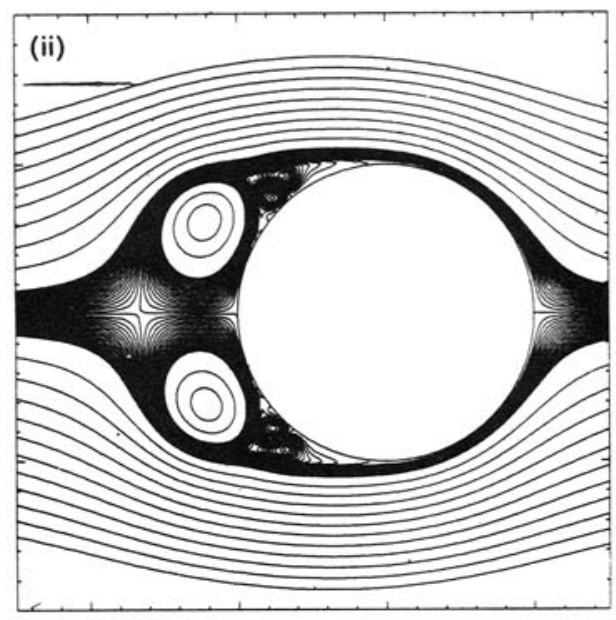

(ii)

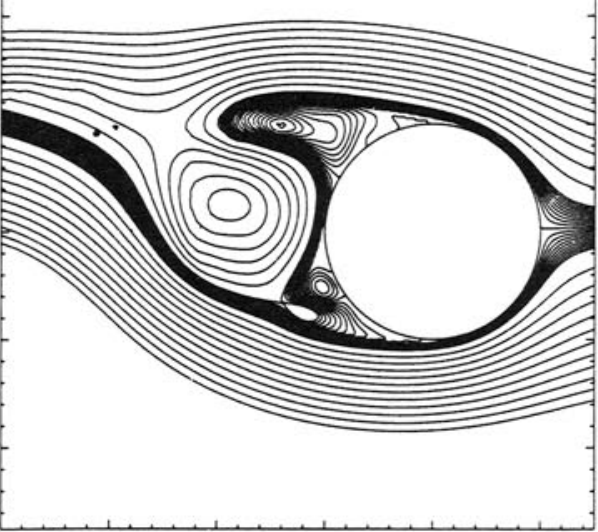

Figure 8. Evolution with time of flow structure in near wake of circular cylinder $(\operatorname{Re}=3000) ;(a)$ in the early stage $t=3.0$ (i), 4.0 (ii), (b) in a long time period, $t=30$ (i), 35 (ii), $39 \cdot 5$ (iii). 
with different scales, i.e. the primary vortex with a scale of $O(R)$, the secondary vortices generated in the vicinity of the cylinder surface with a small scale of $O(\sqrt{ } v)$, and further secondary vortices near the separated shear layer, with a variable scale, which are similar to the results given by Braza et al (1986). Our results exhibit detailed interaction processes among these vortices, such as the growth and merging of secondary vortices forming finally a new primary vortex. Results also show the periodic generation and disappearance of secondary vortices near the body surface. Figures $8 \mathrm{a}$ and $\mathrm{b}$ show some examples of the evolution of vortex structure in the near wake in the early stages and over long time periods of the flow respectively. The calculated flow features, such as the separation angles, the radial velocity component behind the cylinder, the drag and the Strouhal numbers, are compared with many previous measurements and pure numerical solutions of the $\mathrm{N}-\mathrm{S}$ equations (e.g. Ta Phuoc Loc \& Bouard 1985), respectively, and satisfactory agreement is obtained. The numerical simulation of separated flow about the cylinder in steady flow at high Reynolds number and with harmonically oscillatory oncoming flow at different $\mathrm{KC}$ and $\beta$ parameters has also been carried out. The instability phenomenon of flow structure containing multiple small-scale vortices in the vicinity of the cylinder surface is simulated, the vorticity distribution near the cylinder and the force on the cylinder are obtained and compared with some previous results from Bouard \& Coutanceau (1980) and Obasaju et al (1988) respectively. Our results also show that the present method has the advantage of the finite-difference method for the solution of $\mathrm{N}-\mathrm{S}$ equations in accurately predicting the fine structure of the flow field, as well as the advantage of the vortex method in efficiently computing the global characteristics of the separated flow. Moreover, it saves computer time.

\section{Three-dimensional evolution of vortex structure and the early features of the coherent structure behind a 2-D circular cylinder}

In this section, the mechanism and dynamic process for the three-dimensional evolution of Kármán vortices and vortex filaments in the braid regions, and the early features of turbulent structure in the wake behind a circular cylinder at moderate and high Reynolds numbers, are studied numerically (Ling et al 1992a; Ling \& Wu 1992). The vortex filaments in the wake flow field are tracked by the inviscid vortex dynamics and the localized-induction approximation (LIA) method. The time-averaged velocity field of the wake, which can be obtained from experimental measurements or simulated numerically by disposing concentrated vortices, and the Kármán vortex street flow field, are considered as two kinds of the background flow. The interaction among the Kárman vortices is considered in the calculation.

The results show that the Kármán vortex and the vortex filament in the braid region have spanwise instability. Through the interaction between the self-induced motion and the background flow, the initial Kármán vortex in the time-averaged velocity field evolves into a special configuration akin to a "horseshoe-like" vortex (in the inclined plane) in the topological structure of the vorticity field. The upper and lower "horseshoe" vortices lie respectively on planes at approximately $45^{\circ}$ and $135^{\circ}$ to the $x-z$ plane ( $x$ is the streamwise direction and $z$ is the spanwise direction). Figure 9a shows examples of evolution with time of the Kármán vortices. An initial spanwise vortex filament at the saddle point of the braid region is turned gradually to the plane of the principal strain and is stretched. Eventually this vortex filament 
(a)
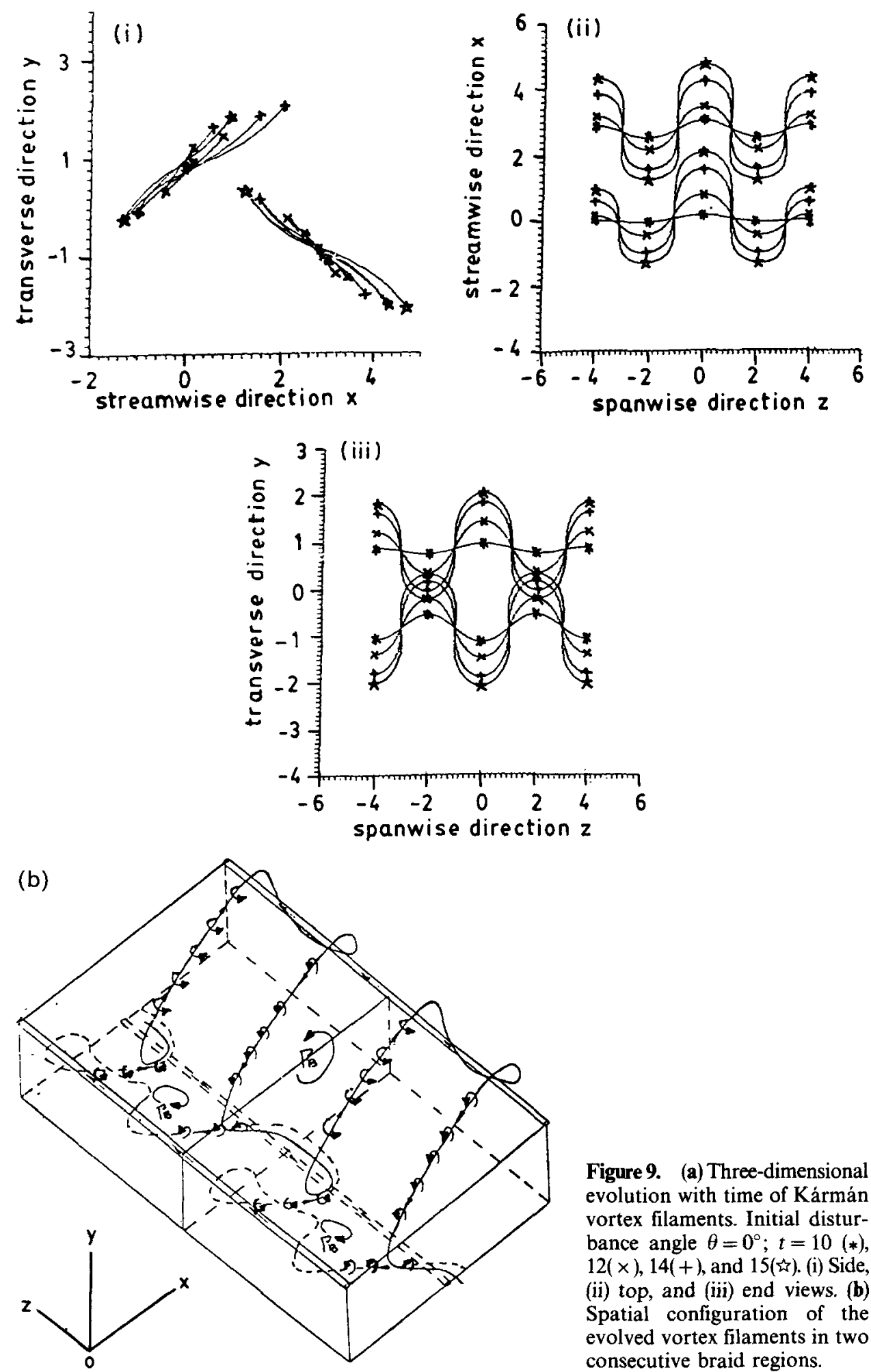

Figure 9. (a) Three-dimensional evolution with time of Kármán vortex filaments. Initial disturbance angle $\theta=0^{\circ} ; t=10(*)$, $12(x), 14(+)$, and 15 (i) . (i) Side, (ii) top, and (iii) end views. (b) Spatial configuration of the evolved vortex filaments in two consecutive braid regions. 
connects with two consecutive Kármán vortices and forms a closed vortex loop. The alternating sign vortex loops in two consecutive braid regions are all aligned in the streamwise direction. Depending on the orientation of the initial disturbance, the closed vortex loops along the spanwise direction show either a symmetric or an asymmetric configuration. Figure $9 \mathrm{~b}$ shows the spatial configuration of the evolved vortex filaments in two consecutive braid regions and the closed vortex loops for the asymmetric configuration. Our calculations present the temporal and spatial processes for the reorientation and the redistribution of the initial spanwise vorticity field, and the streamwise vorticity arises from natural instability. It demonstrates that the deformation and stretching of Kármán vortices and the vortex filaments in the braid region lead to three-dimensional vortex systems which consist of spatial large scale "horseshoe" vortices and three-dimensional small scale vortex loops. They exhibit the early features of the coherent structure of turbulent flow in wake development. The mechanism of the formation of 3-D structure based on present results is different than that in the numerical model of Okubo et al (1988), where a 3-D structure is formed only by deformation of Kármán vortices, but is akin to the suggestion of Williamson (1988) where the braid region effect is stressed. However, the present results are, in some aspects, in agreement with the previous experimental flow visualization. The calculated global features of the structures are not related to the orientation of the initial small disturbance.

This study was supported by the National Natural Science Foundation of China and the Laboratory for Nonlinear Mechanics, Institute of Mechanics, Chinese Academy of Sciences, as well as by the National Basic Research project "Nonlinear Science".

\section{References}

Bouard R, Coutanceau M 1980 The early stage of development of the wake behind an impulsively started cylinder for $40<\operatorname{Re}<10^{4}$. J. Fluid Mech. 101: 583-608

Bradbury L J S 1976 Measurements with a pulsed-wire and a hot-wire anemometer in highly turbulent wake of a normal flat plate. J. Fluid Mech. 77: 493-497

Braza M, Chassaing P, Minh H H 1986 Numerical study and physical analysis of the pressure and velocity fields in the near wake of a circular cylinder. J. Fluid Mech. 165: 79-130

Chein R, Chung J N 1988 Discrete-vortex simulation of flow over inclined and normal plates. Comput. Fluids 16: 405-427

Keulegan C H, Carpenter L H 1958 Forces on cylinders and plates in an oscillating fluid. $J$. Res. Natl. Bur. Stand. 60: 323-339

Kiya M, Arie M 1980 Discrete-vortex simulation of unsteady separated flow behind a nearly normal plate. Bull. JSME 23: 1451-1458

Lian W 1980 A numerical study of two dimensional separated flow past bluff bodies at moderate KC numbers. Appl. Ocean Res. 10: 114-119

Ling G C, Guo L, Wu Z B, Ma H Y 1992a Preliminary study of the three-dimensional deformation of the vortex in Karman vortex street. Acta Aerodyn. Sin. 10:90-97 (in Chinese)

Ling G C, Ling G P, Wang Y P 1992b Domain decomposition hybrid method for numerical simulation of bluff body flows. Sci. China (Sci. Sin) A35: 977-990 (No. 1, pp. 70-79, in Chinese)

Ling G C, Liu G H 1992 Flow structures and force characteristics for flat plate in oscillatory flows with $\mathrm{KC}$ number from 2 to 40 and in combined flows. Acta Mech. Sin. 8: 35-43

Ling G, Luo C M 1991 Investigations of wake flows of a flat plate in steady, oscillatory and combined flows. Acta Mech. Sin. 7: 21-30 
Ling G C, Wu Z B 1992 Numerical study on the three-dimensional evolution of the vortex and the early features of coherent structure in the wake behind a circular cylinder. Proc. Int. Conf. on Fluid Mechanics and Theoretical Physics, June, Beijing, China, pp. 85-86

Obasaju E D, Bearman P W, Graham J M R 1988 A study of forces, circulation and vortex patterns around a circular cylinder in oscillating flow. J. Fluid. Mech. 196: 467-494

Okubo M, Yamane R, Oshima S 1988 Coherent structure in the turbulent wake behind a circular cylinder. 2. Numerical simulation using the vortex filament model. Fluid Dyn. Res. 4: $39-46$

Ongoren A, Rockwell D 1988 Flow structure from an oscillating cylinder. J. Fluid Mech. 191: 197-223, 225-245

Sarpkaya T 1986 Force on a circular cylinder in viscous oscillatory flow at low KeuleganCarpenter numbers. J. Fluid Mech. 165: 61-71

Ta Phuoc Loc, Bouard R 1985 Numerical solution of the early stage of the unsteady viscous flow around a circular cylinder: a comparison with experimental visualization and measurements. J. Fluid Mech. 160: 93-117

Williamson C H K 1988 The existence of two stages in the transition to three dimensionality of a cylinder wake. Phys. Fluids 31: 3165-3168

Williamson C H K 1992 The natural and forced formation of spot-like 'vortex dislocations' in the transition of a wake. J. Fluid Mech. 243: 393-442 